\title{
Propriedades físicas da madeira de Calycophyllum spruceanum Benth. em função do diâmetro e da posição (base e topo) no fuste
}

\author{
Physical properties of Calycophyllum spruceanum Benth. wood \\ according on the diameter and position (base and top) in stem
}

\author{
Breno Henrique Pedroso de Araújo', Madson Alan Rocha de Sousa ${ }^{2}$, \\ Henrique Eduardo Mendonça Nascimento ${ }^{3}$, Antônio José Vinha Zanuncio ${ }^{4}$, \\ Danielle Miranda de Sousa Rodrigues ${ }^{5}$ e Marcelino Carneiro Guedes ${ }^{6}$
}

\begin{abstract}
Resumo
O conhecimento das propriedades tecnológicas da madeira é fundamental para o manejo e uso racional das espécies florestais. $O$ estudo das propriedades físicas da madeira de Calycophyllum spruceanum teve por objetivo fornecer subsídios para a produção de madeira em floresta de várzea estuarina. Foram coletadas amostras de madeira na base e topo do fuste de 15 árvores com diferentes diâmetros para a determinação das propriedades físicas (densidade básica, fator anisotrópico e contrações axial, radial, tangencial e volumétrica), conforme norma NBR 7190/97. A madeira de $C$. spruceanum apresentou média geral de $0,65 \mathrm{~g}_{\mathrm{cm}} \mathrm{cm}^{-3}$ para densidade básica, 5,1\% para contração radial, 7,3\% para contração tangencial, $14,1 \%$ para contração volumétrica e 1,6 para fator anisotrópico. Pela análise de covariância verificou-se efeito significativo da posição no fuste apenas para fator anisotrópico e contração tangencial, em que os maiores valores para estas propriedades foram obtidos na base do fuste das árvores. A inexistência de efeito significativo do diâmetro à altura do peito (DAP) das árvores sobre as propriedades físicas estudadas, associada aos valores de densidade básica e adequados, sob o aspecto da estabilidade dimensional, para o fator anisotrópico evidenciam o potencial madeireiro da espécie, mesmo proveniente de indivíduos menores que o diâmetro mínimo de corte $(50 \mathrm{~cm})$, estabelecido pela legislação brasileira.
\end{abstract}

Palavras-chave: densidade básica, floresta de várzea, pau-mulato, qualidade da madeira, retratibilidade.

\begin{abstract}
The knowledge of the technological properties of the wood is important for the management and rational use of forest species. The study of the physical properties of Calycophyllum spruceanum wood aims to provide subsidies for timber production in estuarine floodplain forests. Samples were collected at base and top of the stem from 15 trees with different diameters to determine the physical properties (basic density, anisotropic factor and axial, radial, tangential and volumetric shrinkage), according to NBR 7190/97. C.

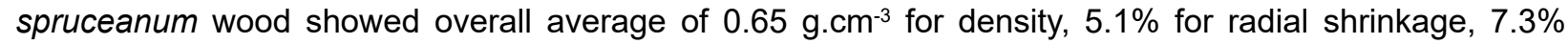
for tangential shrinkage, $14.1 \%$ for volumetric shrinkage and 1.6 for anisotropic factor. By analysis of covariance there was a significant effect of position on the stem only for anisotropic factor and tangential shrinkage, where the highest values for these properties were obtained at the base of the stem of the trees. The inexistence of significant effect of diameter at breast height (DBH) trees on the physical properties studied associated with values of basic density and adequate, from the aspect of dimensional stability for the anisotropic factor show that the timber of the species presents possibilities to be used as solid wood, even from trees under the minimum logging diameters $(50 \mathrm{~cm})$, established by Brazilian law.
\end{abstract}

Keywords: basic density, floodplain forest, pau-mulato, wood quality, retractibility.

\footnotetext{
${ }_{1}^{1}$ Professor na área de Recursos Naturais. IFAP - Instituto Federal de Educação, Ciência e Tecnologia do Amapá. Rua Nilo Peçanha, 1263, Bairro Cajari - 68.920-000 - Laranjal do Jari, AP, Brasil. E-mail: breno.araujo@ifap.edu.br.

2Professor Assistente. UEPA - Universidade do Estado do Pará. Rodovia PA, s/n, Bairro Angelim - 68.625-000 - Paragominas, PA, Brasil. E-mail: madsonalan@ueap.br.

3Pesquisador Associado. INPA - Instituto Nacional de Pesquisas da Amazônia. Av. André Araújo, 2.936 - Caixa Postal 2223 - 69067-375 - Manaus, AM, Brasil. E-mail: hnasci@gmail.com.

${ }^{4}$ Professor colaborador. Unicentro - Universidade Estadual do Centro-Oeste. PR 153, Km 7, s/n, Bairro Riozinho - 84500000 - Irati, PR, Brasil. E-mail: ajvzanuncio@yahoo.com.br.

${ }^{5}$ Graduada em Engenharia Florestal. UEAP - Universidade do Estado do Amapá. Av. Presidente Vargas, 650, Bairro Central, Km 5, 2600 - 68.900-070 - Macapá - AP, Brasil. E-mail: dani enflor23@yahoo.com.br.

${ }^{6}$ Pesquisador, Doutor. Embrapa Amapá. Rodovia Juscelino Kubitschek, Km 5, 2600 - 68903-4 - Macapá - AP, Brasil. E-mail: marcelino.guedes@embrapa.br
} 
Araújo et al. - Propriedades físicas da madeira de Calycophyllum spruceanum Benth.

em função do diâmetro e da posição (base e topo) no fuste

\section{INTRODUÇÃO}

As propriedades físicas da madeira são importantes para definir sua melhor utilização industrial e inseri-las no mercado, reduzindo a demanda por espécies cujas reservas estejam se exaurindo (GOMES et al., 2011; MIRANDA et al., 2012).

O comportamento físico da madeira varia entre espécies, entre indivíduos da mesma espécie e dentro de um único fuste no sentido medula-casca e da base para o topo (MELO et al., 2014; LIMA; GARCIA, 2010; COUTO et al., 2012), sendo influenciado pela idade, taxa de crescimento, tratamentos silviculturais e sítio (KOLLMANN; CÔTÉ, 1968; LONGUI et al., 2012; MIRANDA et al., 2012; SOTELO MONTES et al., 2007). Entender esta variação é necessário para definir o diâmetro mínimo de corte, permitir o manejo específico e aumentar o número de espécies passíveis de exploração, respeitando os processos ecológicos que garantem a manutenção dos táxons e de seus estoques.

A densidade é o principal índice de qualidade da madeira, devido à sua relação com as demais propriedades (COUTO et al., 2012) e por isso, é uma das características mais estudadas. Em geral, madeiras com alta densidade apresentam maior variação volumétrica, devido a maior quantidade de água de impregnação na parede celular por unidade de volume. Quando em processo de secagem abaixo do ponto de saturação das fibras, a perda desse tipo de água ocasiona acentuada contração (SILVA; OLIVEIRA, 2003; GLASS; ZELINKA, 2010). Além disso, madeiras de alta densidade apresentam maior resistência mecânica, sendo recomendadas para uso estrutural (LIMA et al., 2014).

Alguns estudos comprovam não haver um padrão único na variação de densidade básica. Panshin e De Zeew (1980); Downes e Raymond (1997) apresentaram alguns modelos de variação dessa propriedade ao longo do tronco das árvores. No Brasil, estudos dessa natureza são predominantes para as espécies dos gêneros Eucalyptus e Pinus. No entanto, pesquisas relacionadas às espécies nativas vêm crescendo, como exemplo destacam-se os trabalhos de Longui et al. (2010); Lima et al. (2011a); Valente et al. (2013); Delucis et al. (2013).

Nesse contexto, o pau-mulato (Calycophyllum spruceanum Benth.), pertencente à família Rubiaceae, é uma espécie nativa com elevada densidade em florestas secundárias no estuário amazônico (JONG, 2001; ALMEIDA, 2004), sua madeira de excelente qualidade tem grande importância econômica na região (QUEIROZ; MACHADO, 2007). No Amapá, representa aproximadamente 25\% $\left(16.508 \mathrm{~m}^{3}\right)$ do volume de madeira comercializado e contribui com $21 \%$ ( $\left.\mathrm{R} \$ 1.140 .858,00\right)$ da receita bruta, sendo comercializada como tábuas, caibros, flexais, esteios e vigas nas microsserrarias em várzeas estuarinas (GUEDES et al., 2012; CASTILHO, 2013). No Peru, C. spruceanum é utilizada na produção de lenha e carvão, devido ao alto poder calorífico (SOTELO MONTES et al., 2003). Esta espécie possui copa pequena e rala, sendo opção em sistemas agroflorestais, pois permite a passagem de luz e causa pouco sombreamento.

Na região amazônica estuarina é frequente encontrar microsserrarias familiares que exploram e processam a madeira de C. spruceanum de forma tradicional, visando à produção de peças para construção civil, principalmente. Existe ainda, a utilização de seus resíduos como lenha para cocção de alimentos, bem como o uso de fustes de pequenos diâmetros na construção de galpões para armazenagem de ferramentas e da produção agrícola familiar.

Em plantios de C. spruceanum com idade de 16, 28 e 39 meses (SOTELO MONTES et al., 2006; 2007) e aos 32 meses (WEBER; SOTELO MONTES, 2005) foi evidenciado, com base na avaliação das propriedades tecnológicas, o potencial da espécie em produzir madeira em idades jovens. Por sua vez, Gutiérrez e Ramos (2013) identificaram o potencial da espécie para estudos dendrocronológicos na Amazônia peruana, destacando que os anéis de crescimento são delimitados por zonas fibrosas mais estreitas radialmente.

Os estudos de caracterização física da madeira de C. spruceanum se justificam devido a poucas informações científicas sobre as suas propriedades tecnológicas e pela necessidade do maior conhecimento das madeiras nativas do Brasil. Diante disso, este trabalho teve como objetivo avaliar as propriedades físicas da madeira de C. spruceanum em função do diâmetro e diferentes posições no fuste (base e topo), com o intuito de viabilizar o aproveitamento da regeneração natural em florestas de várzea por meio do uso da madeira de árvores com DAP menores que $50 \mathrm{~cm}$. 


\section{MATERIAL E MÉTODOS}

\section{Caracterização da área de estudo}

O estudo foi realizado em floresta de várzea do estuário amazônico, no município de Mazagão, estado do Amapá $\left(00^{\circ} 14\right.$ > 35»S e $51^{\circ} 22$ 2 59» $\left.\mathrm{O}\right)$, em um afluente do rio Amazonas denominado de igarapé Bispo. O clima da região é do tipo Ami, equatorial superúmido, conforme a classificação de Köppen. A temperatura média mínima é de $23^{\circ} \mathrm{C}$ e a média máxima de $38^{\circ} \mathrm{C}$, com umidade relativa média de $80 \%$. A precipitação pluviométrica anual oscila em torno de $2.500 \mathrm{~mm}$ (GAZEL FILHO et al., 2008). O solo é classificado como Gleissolo Háplico (SANTOS; TARDIN, 2003). A vegetação predominante da região é a Floresta Ombrófila Densa Aluvial, também conhecida por floresta de várzea (IBGE, 2012).

O local de coleta consiste em uma floresta secundária de várzea que foi abandonada pela agricultura itinerante, onde posteriormente houve abundante regeneração de C. spruceanum. O local está situado dentro da Reserva Extrativista Rio Cajari. Em cumprimento ao que determina a Instrução Normativa $N^{\circ}$ 03/2014 do Instituto Chico Mendes de Conservação da Biodiversidade (ICMBio), foi solicitada uma Autorização para atividades com finalidade científica. A Embrapa Amapá, por meio do Projeto Florestam "Ecologia e Manejo Florestal para Uso Múltiplo de Várzeas do Estuário Amazônico" obteve a referida autorização (n 29982-2, código de autenticação 93489853 e data de emissão em 07/08/2011.) para realização da coleta e transporte das amostras de madeira.

\section{Delineamento amostral}

Para a coleta das amostras de madeira foram derrubadas 15 árvores de C. spruceanum, com diâmetro à altura do peito (DAP) de 18,26, 38, 45 e $55 \mathrm{~cm}$, sendo três repetições (árvores) para cada diâmetro. A altura total de cada árvore foi de 20,0;23,0;26,5;24,1;21,2;23,4;25,0;26,0;21,1; 29,$0 ; 25,0 ; 25,0 ; 26,1 ; 25,0$ e $21,3 \mathrm{~m}$.

De cada árvore derrubada foram retiradas duas toras de 1,20 $\mathrm{m}$ de comprimento, sendo uma da base (20 cm acima do solo) e uma no topo (últimos 1,20 $\mathrm{m}$ da altura comercial do fuste). A altura comercial foi considerada até a inserção de galhos. De cada uma dessas toras foram seccionadas pranchas diametrais, com dimensões de $10 \mathrm{~cm}$ em espessura e 1,20 m de comprimento e largura variando em função do diâmetro de cada árvore. A partir dessas pranchas foram retirados corpos de prova para realização de testes físicos (Figura 1).
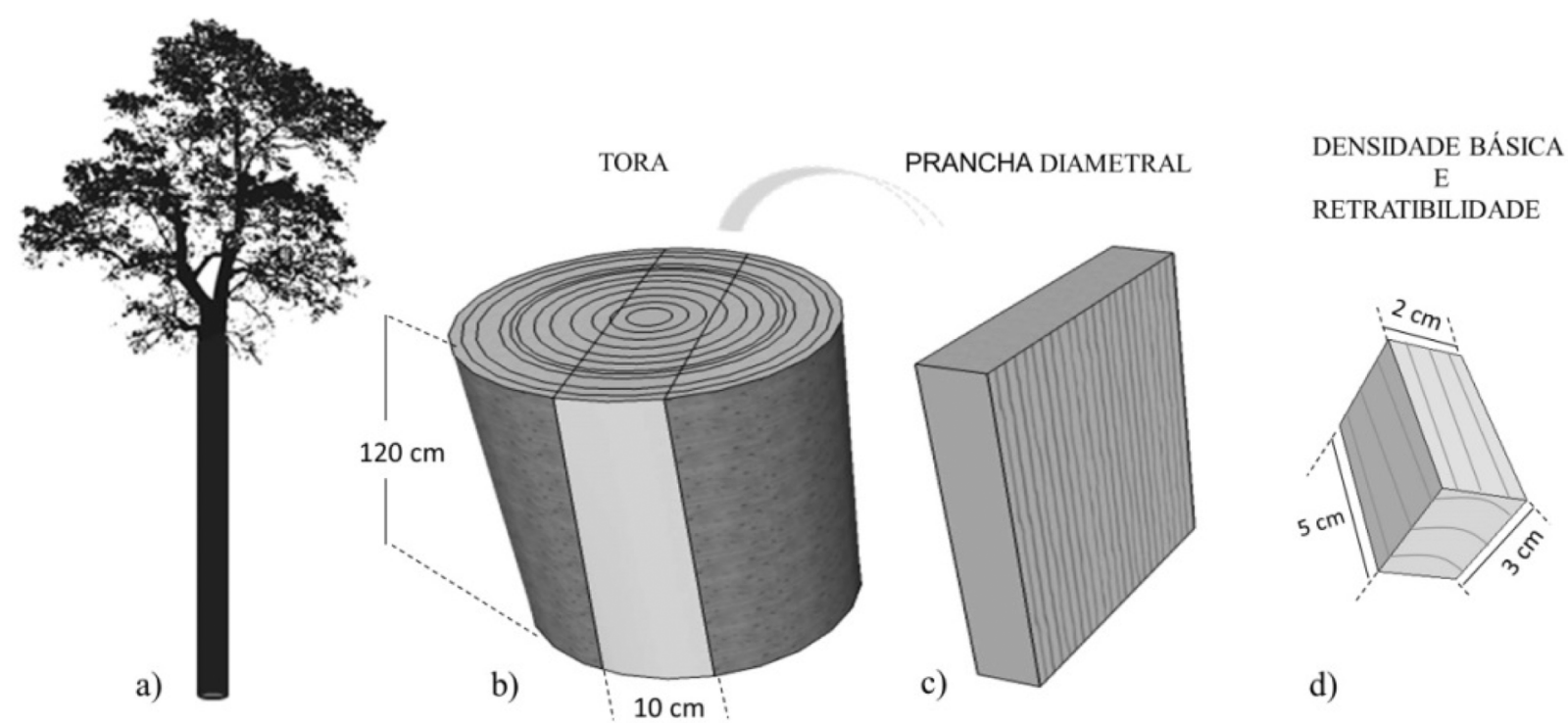

Figura 1. Representação esquemática da amostragem de madeira de C. spruceanum. a) Árvore em pé; b) Tora retirada da base ou topo; c) Prancha diametral; d) Corpos de prova para ensaios de densidade básica e retratibilidade (Foto: Breno Araújo, 2012).

Figure 1. Diagram showing the sampling of $C$. spruceanum wood. a) Tree standing; b) Log removed from the base or top; c) Diametrical board; d) Samples for basic density tests and shrinkage. 
Araújo et al. - Propriedades físicas da madeira de Calycophyllum spruceanum Benth.

em função do diâmetro e da posição (base e topo) no fuste

\section{Caracterização física da madeira}

A densidade básica e a retratibilidade (contrações axial, radial, tangencial e volumétrica) foram determinadas em corpos de prova com dimensões de $2 \mathrm{~cm}$ x $3 \mathrm{~cm}$ x $5 \mathrm{~cm}$, conforme procedimentos da NBR 7190 (ABNT, 1997). Foram confeccionados seis corpos de prova de cada árvore (três repetições da base e três do topo do fuste), totalizando 90 corpos de prova. O fator anisotrópico foi obtido pela relação entre a contração tangencial e a radial.

Os corpos de prova foram orientados em função do plano de corte (axial, radial e tangencial) e saturados por meio de tratamento em sistema com recipiente de vidro e bomba a vácuo, por 120 horas. Após a saturação, foram medidas as dimensões nos respectivos planos de corte, com o auxílio de paquímetro digital com precisão de $0,01 \mathrm{~mm}$ para a obtenção do volume da madeira saturada. Posteriormente, os mesmos foram secos em estufa a $105 \pm 3{ }^{\circ} \mathrm{C}$, até alcance de massa constante, para determinação da massa seca e do volume da madeira seca, utilizando-se uma balança com precisão de 0,01 g e paquímetro digital, respectivamente.

\section{Análise de dados}

A análise de covariância (ANCOVA) foi realizada para testar os efeitos do DAP e posição no fuste sobre a densidade básica, contrações da madeira e fator anisotrópico. A ANCOVA considerou a posição no fuste (base e topo) como um fator fixo e o DAP como a covariável. As análises foram realizadas no Statistica 10.0 (STATSOFT, 2012).

\section{RESULTADOS E DISCUSSÃO}

A média da densidade básica foi de 0,65 g.cm ${ }^{-3}$, considerando os valores das amostras da base e do topo do fuste de C. spruceanum. Esta propriedade apresentou os menores valores para coeficiente de variação, destacando-se como a mais estável entre as fontes de variação consideradas (Tabela 1). Sotelo Montes et al. (2007) estudando madeira jovem (32 meses de idade) de C. spruceanum também encontraram coeficientes de variação baixos, entre 6 e 7,6\% para a densidade básica.

Tabela 1. Valores médios das propriedades físicas da madeira de C. spruceanum.

Table 1. Mean values of physical properties of the C. spruceanum wood.

\begin{tabular}{|c|c|c|c|c|c|c|c|c|c|c|c|}
\hline \multirow{2}{*}{$\begin{array}{l}\text { DAP } \\
(\mathrm{cm})\end{array}$} & \multirow{2}{*}{$\begin{array}{l}\text { Posição } \\
\text { (Fuste) }\end{array}$} & \multicolumn{2}{|c|}{$\begin{array}{c}\text { Densidade } \\
\text { Básica }\left({\left.\mathrm{g} . \mathrm{cm}^{-3}\right)}^{2}\right.\end{array}$} & \multicolumn{2}{|c|}{$\begin{array}{c}\text { Contração } \\
\text { Volumétrica (\%) }\end{array}$} & \multicolumn{2}{|c|}{$\begin{array}{c}\text { Contração Ra- } \\
\text { dial (\%) }\end{array}$} & \multicolumn{2}{|c|}{$\begin{array}{c}\text { Contração } \\
\text { Tangencial (\%) } \\
\end{array}$} & \multicolumn{2}{|c|}{$\begin{array}{c}\text { Fator } \\
\text { Anisotrópico }\end{array}$} \\
\hline & & Média & CV (\%) & Média & CV (\%) & Média & CV (\%) & Média & CV (\%) & Média & CV (\%) \\
\hline 18 & Base & 0.65 & 5.86 & 16.76 & 25.16 & 6.19 & 32.69 & 8.13 & 15.23 & 1.43 & 16.88 \\
\hline 18 & Topo & 0.62 & 4.92 & 12.31 & 7.87 & 5.20 & 3.11 & 5.47 & 13.55 & 1.01 & 15.31 \\
\hline 26 & Base & 0.65 & 7.43 & 15.33 & 9.64 & 5.48 & 18.87 & 7.74 & 7.81 & 1.52 & 21.55 \\
\hline 26 & Topo & 0.63 & 7.19 & 12.72 & 13.89 & 4.85 & 23.84 & 6.50 & 8.00 & 1.51 & 27.10 \\
\hline 38 & Base & 0.70 & 2.55 & 13.32 & 6.73 & 4.32 & 30.74 & 7.59 & 10.85 & 2.28 & 33.89 \\
\hline 38 & Topo & 0.67 & 5.99 & 13.01 & 25.18 & 5.00 & 19.54 & 6.46 & 22.44 & 1.58 & 19.39 \\
\hline 45 & Base & 0.65 & 4.26 & 13.44 & 14.53 & 4.40 & 15.88 & 7.39 & 15.12 & 1.98 & 3.46 \\
\hline 45 & Topo & 0.65 & 5.75 & 16.52 & 11.48 & 5.56 & 17.25 & 8.53 & 5.13 & 1.58 & 14.47 \\
\hline 55 & Base & 0.66 & 2.73 & 14.93 & 7.66 & 5.10 & 10.38 & 8.02 & 3.74 & 1.66 & 8.44 \\
\hline 55 & Topo & 0.64 & 4.01 & 14.25 & 13.81 & 4.86 & 9.39 & 7.39 & 11.51 & 1.54 & 5.38 \\
\hline
\end{tabular}

A média da densidade básica de C. spruceanum encontra-se de acordo com o resultado obtido por Woodcock (2000). Em contrapartida, é considerada relativamente baixa, quando comparada aos resultados de Acevedo e Kikata (1994) e Sotelo Montes et al. (2007), que estudaram a madeira dessa espécie na Amazônia peruana e encontraram densidade básica de 0,76 e 0,71 (g.cm-3), respectivamente.

A densidade é uma das propriedades mais importantes da madeira, pois é a que melhor expressa sua qualidade para fins tecnológicos e industriais, contribuindo para indicar o uso mais adequado para cada espécie. Ao comparar a densidade básica média da madeira de C. spruceanum com valores de espécies utilizadas comercialmente, como o Brosimum parinarioides $\left(0,59 \mathrm{~g} . \mathrm{cm}^{-3}\right)$, Parkia paraensis $\left(0,56{\mathrm{~g} . \mathrm{cm}^{-3}}^{-3}\right.$ (SILVEIRA et al., 2013), Carapa guianensis $\left(0,59 \mathrm{~g} . \mathrm{cm}^{-3}\right)$, Goupia glabra $\left(0,68 \mathrm{~g} . \mathrm{cm}^{-3}\right)$ e Vochysia spp. $\left(0,65{\left.\mathrm{~g} . \mathrm{cm}^{-3}\right)}^{-}\right.$(NASCIMENTO et al., 1997) verifica-se que C. spruceanum apresenta potencial de uso na marcenaria, carpintaria, móveis e construções em geral. 
Os resultados de contração deste trabalho (Tabela 1) são próximos aos encontrados no estudo de Sotelo Montes et al. (2007) que obtiveram valores médios para as contrações tangencial, radial e volumétrica de 7,50\%, 3,48\% e 11,19\%, respectivamente, para a madeira de C. spruceanum aos 36 meses de idade na Amazônia peruana. Comparando a contração volumétrica de madeiras comerciais como Manilkara huberi (17,6\%), Calophyllum brasiliense (16,9\%), Goupia glabra $(16,1 \%)$ e Bowdichia sp. (15,1\%) (ZENID, 2009), observa-se que C. spruceanum apresenta contração volumétrica inferior $(14,26 \%)$, indicando que sua madeira tem menores alterações volumétricas com a perda de água, podendo vir a substituir o uso dessas espécies quando considerado o aspecto de sua estabilidade dimensional.

Pela análise de covariância observou-se que o fator posição no fuste (base e topo) resultou em efeitos significativos apenas para a contração tangencial e fator anisotrópico, enquanto que o fator DAP não gerou efeito significativo sobre as propriedades estudadas (Tabela 2). Tal fato vislumbra a possibilidade de uso da espécie em diâmetros menores que o estabelecido pela legislação (Instrução normativa 05/2006 do IBAMA), garantindo padrões similares de qualidade da madeira em relação aos diâmetros maiores legalmente explorados.

Tabela 2. Efeito da posição no fuste e DAP sobre as propriedades da madeira de Calycophyllum spruceanum (F= F de Fisher; $p=$ valor- $p$ ).

Table 2. Effect of position on the stem and DBH on the wood properties of Calycophyllum spruceanum ( $F=F$ Fisher, $\mathrm{p}=\mathrm{p}$-value).

\begin{tabular}{|c|c|c|c|c|c|c|c|c|c|c|}
\hline \multirow[t]{2}{*}{ FONTE } & \multicolumn{2}{|c|}{$\begin{array}{c}\text { Densidade } \\
\text { básica }\left(\mathbf{g} . \mathrm{cm}^{-3}\right)\end{array}$} & \multicolumn{2}{|c|}{$\begin{array}{l}\text { Contração } \\
\text { radial (\%) }\end{array}$} & \multicolumn{2}{|c|}{$\begin{array}{c}\text { Contração } \\
\text { tangencial (\%) }\end{array}$} & \multicolumn{2}{|c|}{$\begin{array}{c}\text { Contração } \\
\text { volumétrica (\%) }\end{array}$} & \multicolumn{2}{|c|}{$\begin{array}{c}\text { Fator } \\
\text { anisotrópico }\end{array}$} \\
\hline & $\mathbf{F}$ & Valor-p & $\mathbf{F}$ & Valor-p & $\mathbf{F}$ & Valor-p & $\mathbf{F}$ & Valor-p & $\mathbf{F}$ & Valor-p \\
\hline Posiç & 1,63 & 0,21 & 0,002 & 0,96 & 4,38 & $0,04^{*}$ & 0,98 & 0,33 & 4,49 & $0,04^{*}$ \\
\hline DAP & 0,90 & 0,35 & 1,24 & 0,27 & 2,84 & 0,10 & 0,06 & 0,81 & 3,69 & 0,06 \\
\hline
\end{tabular}

(*) significativo ao nível de $5 \%$ de probabilidade de erro.

Embora alguns estudos com amostragem em diferentes posições longitudinais do fuste de Eucalyptus grandis (HASELEIN et al., 2004), Schizolobium amazonicum (COLLI, 2007), Cordia americana e Alchornea triplinervia (TRAUTENMÜLLER et al., 2014) tenham resultado em menores valores para a densidade básica nas porções superiores do tronco, nesta pesquisa não foram observadas diferenças estatísticas na densidade tanto em função da posição no fuste como em função do diâmetro de pau-mulato (Figura 2).

Resultados similares à nossa pesquisa foram obtidos no estudo de Valente et al. (2013) em que as propriedades físicas da madeira de angico-vermelho não apresentaram padrão definido de variação no sentido longitudinal e também não houve diferença significativa das propriedades entre as três primeiras toras. Os padrões de variação mais evidentes foram observados apenas no sentido radial.

Existem formas diversas de variação da densidade no sentido base-topo, destacando que os fatores que contribuem para tal variação perpassam pela idade da árvore, idade cambial, fatores genéticos, a taxa de crescimento das árvores, condições climáticas, tratamentos silviculturais, sítio, lugar de amostragem no tronco (THIBAUT et al., 2001; ALZATE et al., 2005; SETTE JR. et al, 2012, MENESES et al., 2015) e diferenças estruturais, como a nanoestrutura da parede celular (LOURENÇON et al., 2014). Dessa forma, indica-se que para uma melhor compreensão da variação da densidade na madeira de C. spruceanum, os estudos das propriedades físicas sejam associados a pesquisas de variação anatômica e química de sua estrutura celular, buscando melhor correlacionar tais características em função da idade e/ou diâmetro da árvore.

Para a contração tangencial os valores na base dos fustes foram superiores aos do topo, com exceção das árvores com $45 \mathrm{~cm}$ de DAP. Tal diferença não foi meramente ao acaso, conforme estatísticas da ANCOVA (Tabela 2), ou seja, há um efeito significativo do fator posição do fuste sobre a contração tangencial, bem como sobre o fator anisotrópico.

O princípio da contração se deve ao fato de as moléculas de água estarem ligadas por pontes de hidrogênio às microfibrilas dos polissacarídeos que formam a madeira. Quando estas moléculas são forçadas a sair, deixam um espaço, e as forças de coesão tendem a reaproximar as microfibrilas, causando contração da madeira como um todo. Assim, é esperado que as madeiras mais densas, por terem maior concentração de células de paredes mais espessas, absorvam ou percam mais água por unidade de volume e, consequentemente, tendem a expandir ou contrair mais do que aquelas 
Araújo et al. - Propriedades físicas da madeira de Calycophyllum spruceanum Benth.

em função do diâmetro e da posição (base e topo) no fuste

de menor densidade (OLIVEIRA et al., 2010; SERPA et al., 2003; SILVA; OLIVEIRA, 2003; LIMA et al., 2014), fato corroborado com os resultados encontrados para as árvores de C. spruceanum, onde as madeiras mais densas tiveram maiores contrações.

Considerando-se apenas as características físicas da madeira e conforme a classificação de Durlo e Marchiori (1992), a madeira de C. spruceanum oriunda do topo do fuste é considerada de excelente qualidade, pois apresenta fator anisotrópico entre 1,0 e 1,5, enquanto a madeira da base apresenta um pouco mais de instabilidade dimensional, mas que não inviabiliza seu aproveitamento (Figura 2). Tais resultados sugerem a madeira de C. spruceanum como dimensionalmente estável e menos propensa a defeitos, uma vez que quanto menor a relação presente no fator anisotrópico, menor será a tendência ao fendilhamento e empenamento da peça de madeira durante as alterações dimensionais provocadas pela variação higroscópica (OLIVEIRA; SILVA, 2003; OLIVEIRA et al., 2010).
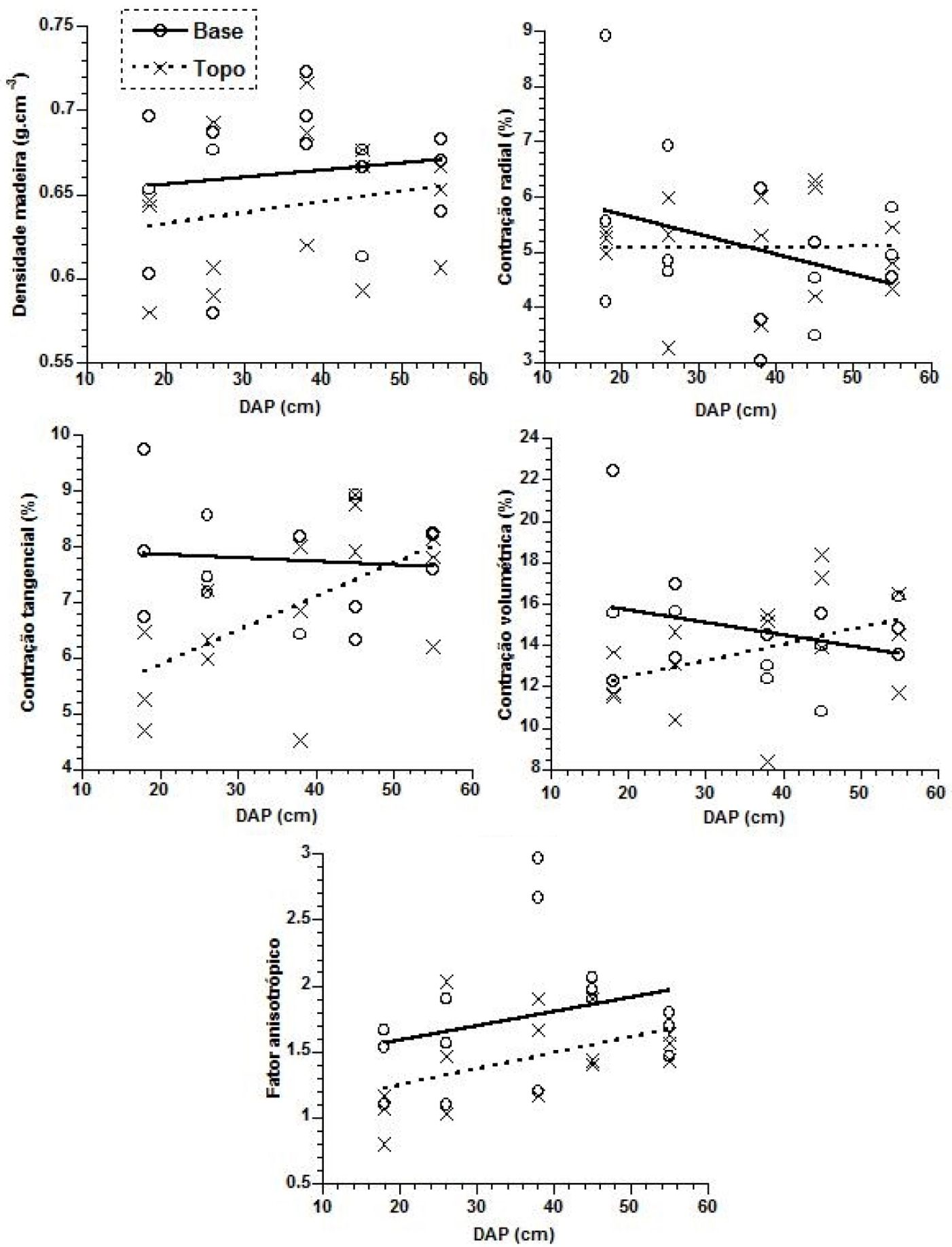

Figura 2. Densidade básica, contrações radial, tangencial e volumétrica e fator anisotrópico para a madeira de Calycophyllum spruceanum em função do DAP e da posição no fuste.

Figure 2. Basic density, radial, tangential and volumetric shrinkage and anisotropic factor for Calycophyllum spruceanum wood according to the dbh and position in the stem. 
A associação da menor movimentação dimensional da madeira com a região do topo do fuste é condicionada pela presença predominante de madeira juvenil. As porções mais altas dos fustes possuem proporcionalmente maiores quantidades de lenho juvenil, formadas nos períodos iniciais de crescimento próximo à medula, caracterizando-se como uma madeira com maiores ângulos microfibrilares da camada $S_{2}$, maior diâmetro do lume e conteúdo de lignina, menor densidade, resistência e rigidez (BENDTSEN, 1978; BALLARIN; PALMA, 2003; VIDAURRE et al., 2011), e ainda menor comprimento, largura, espessura da parede do lume (TOMAZELLO FILHO, 1985).

Em muitas espécies madeireiras a variação das características anatômicas, físicas, mecânicas e químicas, no sentido medula-casca, é bem mais acentuada do que no sentido da base para o topo (CRUZ et al., 2003; LIMA et al., 2011b). Os resultados encontrados para a madeira de C. spruceanum corroboram também com essa tendência, apresentando variação significativa de apenas duas características físicas (contração tangencial e fator anisotrópico) quando comparados base e topo do fuste.

Os resultados das características físicas da madeira de C. spruceanum associados à elevada densidade de regeneração, rápido crescimento e bons valores de incrementos diamétricos encontrados por Guedes et al. (2012) e Castilho (2013), credenciam C. spruceanum como uma espécie potencial para o fornecimento de madeira em curto período de tempo.

\section{CONCLUSÕES}

A madeira de C. spruceanum apresentou boas propriedades físicas, com média de $0,65 \mathrm{~g}_{\mathrm{cm}} \mathrm{cm}^{-3}$ para densidade básica, 5,1\% para contração radial, 7,3\% para contração tangencial, 14,1\% para contração volumétrica e 1,6 para fator anisotrópico.

A inexistência de efeito significativo do diâmetro das árvores de C. spruceanum sobre as propriedades físicas estudadas credenciam a espécie como potencial para uso em diâmetros de corte menores que o estipulado pela legislação vigente, ampliando a oferta desta espécie e favorecendo as populações que vivem do seu manejo. $\mathrm{O}$ fator posição do fuste resultou em efeitos significativos apenas sobre o fator anisotrópico e contração tangencial.

Estudos mais abrangentes das propriedades anatômicas, químicas e mecânicas são necessários para melhor caracterizar o material lenhoso das árvores jovens, visando predizer usos específicos, que possam subsidiar o manejo dessa espécie por comunidades ribeirinhas na Amazônia brasileira.

\section{REFERENCIAS BIBLIOGRÁFICAS}

ABNT - ASSOCIAÇÃO BRASILEIRA DE NORMAS TÉCNICAS. NBR 7190: Projeto de Estruturas de Madeira. Rio de Janeiro, 1997, 107 p.

ACEVEDO, M.; KIKATA, Y. Atlas de Maderas del Perú. Lima: Universidad Nacional Agraria La Molina, 1994.

ALMEIDA, M. C. Pau-mulato da várzea: Calycophyllum spruceanum (Benth.) Hook.f.ex K. Schum. Manaus: INPA, 2004. 2 p. (Informativo Técnico da Rede de Sementes da Amazônia, 6).

ALZATE, S. B. A.; TOMAZELLO FILHO, M.; PIEDADE, S. M. S. Variação longitudinal da densidade básica da madeira de clones de Eucalyptus grandis Hill ex Maiden, E. saligna Sm. e E. grandis $\mathrm{x}$ urophylla. Scientia Forestalis, Piracicaba, n. 68, p. 87-95, 2005.

BALLARIN, A. W.; PALMA, H. A. L. Propriedades de resistência e rigidez da madeira juvenil e adulta de Pinus taeda L. Revista Árvore, Viçosa, v. 27, n. 3, p. 371-380, 2003.

BENDTSEN, B. Properties of wood from improved and intensively managed trees. Forest Products Journal, Madison, v. 28, n. 10, p. 61-72, 1978.

CASTILHO, N. T. Manejo da regeneração natural e produção de madeira de pau-mulato em floresta de várzea do estuário amazônico. 2013. 96 p. Dissertação (Mestrado em Biodiversidade Tropical) - Universidade Federal do Amapá, Macapá, 2013. 
Araújo et al. - Propriedades físicas da madeira de Calycophyllum spruceanum Benth.

em função do diâmetro e da posição (base e topo) no fuste

COLLI, A. Caracterização da madeira de paricá (Schizolobium amazonicum) Huber ex. Ducke e propriedades de chapas de partículas aglomeradas com diferentes porções de fibras de coco (Cocos nucifera L.). 2007. 64 p. Dissertação (Mestrado em Ciência Florestal) - Universidade Federal de Viçosa, Viçosa, 2007.

COUTO, A. M.; PROTÁSIO, T. P.; REIS, A. A.; TRUGILHO, P. P. F. Amostragens longitudinais alternativas para determinação da densidade básica em clones de Eucalyptus sp. Floresta e Ambiente, Seropédica, v. 19, n. 2, p. 184-193, 2012.

CRUZ, C. R.; LIMA, J. T.; MUNIZ, G. I. B. Variações dentro das árvores e entre clones das propriedades físicas e mecânicas da madeira de híbridos de Eucalyptus. Scientia Forestalis, Piracicaba, n. 64, p. 33-47, 2003.

DELUCIS, R. A.; GATTO, D. A.; STANGERLIN; D. M.; CADEMARTORI, P. H. G.; WEINERT, L. C.; VEGA, R. A. Segregação e variação radial de propriedades físicas da madeira juvenil e adulta de cedro (Cedrela fissilis Vellozo). Scientia Forestalis, Piracicaba, v. 41, n. 100, p. 549-556, 2013.

DOWNES, G. M.; RAYMOND, C. A. Variation in wood density in plantation eucalypts. In: DOWNES, G. M.; HUDSON, I. L.; RAYMOND, C. A.; DEAN, G. H.; MICHELL, A. J.; SCHIMLECK, L. R.; EVANS, R.; MUNERI, A. Sampling plantation eucalypts for wood and fibre properties. Melbourne: CSIRO Publishing, p. 88-99, 1997.

DURLO, M. A.; MARCHIORI, J. N. C. Tecnologia da madeira: retratibilidade. Santa Maria: CEPEF/FATEC, 1992. 33 p. (Série Técnica, 10).

GAZEL FILHO, A. B.; YARED, J. A. G.; MOURÃO JR., M.; SILVA, M. F.; CARIM, M. J. V.; JARDIM, M. A. G.; MEDEIROS, T. D. S. Composição florística e estrutura de floresta de várzea no município de Mazagão, estado do Amapá, Brasil. Scientia Forestalis, Piracicaba, v. 36, n. 79, p. 191-201, 2008.

GLASS, S.; ZELINKA; S. L. Moisture relations and physical properties of wood. In: ROSS. R. (Ed.) Wood Handbook: Wood as an Engineering Material. Madison: UDSA Forest Service, 2010. p.4-1-4-19. (FPL General Technical Report, 190).

GOMES, A. R. S.; CAMARGOS, J. A. A.; GONÇALES, J. C.; SANTOS, L. C. Espécies alternativas de madeiras para a indústria moveleira. Revista da Madeira. Curitiba, n. 128, 2011.

GUEDES, M. C.; CASTILHO, N. T. F.; RODRIGUES, D. M. S.; FISCHER, G.; SOUSA, M. ZAHARYA, N.; MOCHIUTTI, S. Regeneração do pau-mulato: Manejo para uso sustentável da várzea. Macapá: Embrapa Amapá, 2012. 2 p.

GUTIÉRREZ, L. A. B.; RAMOS, G. M. V. Anatomia de anillos de crecimiento de 80 especies arbóreas potenciales para estúdios dendrocronológicos em la Selva Central, Perú. Revista de Biología Tropical, San José, v. 61, n. 3, p. 1025-1037. 2013.

HASELEIN, C. R.; LOPES, M. C.; SANTINI, E. J.; LONGHI, S. J.; ROSSO, S.; FERNANDES, D. L. G.; MENEZES, L. F. Características tecnológicas da madeira de árvores matrizes de Eucalyptus grandis. Ciência Florestal, Santa Maria, v. 14, n. 2, p. 145-155, 2004.

IBGE - INSTITUTO BRASILEIRO DE GEOGRAFIA E ESTATÍSTICA. Manual técnico da vegetação brasileira. 2.ed. Rio de Janeiro, 2012. 271 p.

JONG, W. Tree and forest management in the floodplains of the Peruvian Amazon. Forest Ecology and Management, Amsterdam, v. 150, n. 1-2, p. 125-134, 2001.

KOLLMANN, F. F. P.; CÔTÉ, W. A. Principles of wood science and technology. New York: Springer, 1968. 592 p. 
LIMA, I. L.; LONGUI, E. L.; FREITAS, M. L. M.; ZANATTO, A. C. S.; ZANATA, M.; FLORSHEIM, S. M. B.; BORTOLETTO JR., G. Physical-mechanical and anatomical characterization in 26-year-old Eucalyptus resinifera wood. Floresta e Ambiente, Seropédica, v. 21, n. 1, p. 91-98, 2014.

LIMA, I. L.; LONGUI, E. L.; GARCIA, R.; LUCA, E. F.; SILVA JR., F. G.; FLORSHEIM, S. M. B. Propriedades da Madeira de Eucalyptus umbra R. T. Baker em função do diâmetro e da posição radial na tora. Floresta e Ambiente, Seropédica, v. 18, n. 3, p. 289-298, 2011 a.

LIMA, I. L.; PIMENTEL, M. M.; GARCIA, J. N. Propriedades Mecânicas e Densidade Aparente da Madeira de Tectona grandis Linn. F. (Teca) em Função do Espaçamento e da Posição Radial na Tora. Silva Lusitana, Lisboa, v. 19 , n. 2, p. 2241-232, 2011 b.

LIMA, I. L.; GARCIA J. N. Variação da densidade aparente e resistência à compressão paralela às fibras em função da intensidade de desbaste, adubação e posição radial em Eucalyptus grandis Hill ex-Maiden. Revista Árvore, Viçosa, v. 34, n. 3, p. 551-559, 2010.

LONGUI, E. L.; LIMA, I. L.; FLORSHEIM, S. M. B.; MELO, A. C. G.; ROMEIRO, D.; SUCKOW, I. M. S.; TESTONI, L. N. Estrutura do lenho de Plathymenia reticulata e algumas implicações na eficiência hidráulica e resistência mecânica. Floresta, Curitiba v. 42, n. 2, p. 335-346, 2012.

LONGUI, E. L.; LIMA, I. L.; SILVA JR., F. G. S.; BUFOLO, A.; SUCKOW, I. M. S.; FLORSHEIM, S. M. B. Variação radial das características anatômicas, densidade aparente, teores de extrativos, lignina e holocelulose na madeira de Piptadenia gonoacantha (Mart.) Macbr. Scientia Forestalis, Piracicaba, v. 38, n. 87, p. 341-353, 2010.

LOURENÇON, T. V.; MATTOS, B. D.; GATTO, D. A.; BULIGON, E. A.; HASELEIN, C. R. Determinação da idade de transição entre lenho juvenil e lenho adulto para três espécies florestais por meio de suas propriedades mecânicas. Floresta e ambiente, Seropédica, v. 21, n. 2, p. 251-260, 2014.

MELO, L. E. L.; SILVA, C. J.; PROTÁSIO, T. P. TRUGILHO, P.F.; SANTOS, I.S.; URBINATI, C. V. Influence of spacing on some physical properties of Schizolobium parahyba var. amazonicum (Huber ex Ducke). Scientia Forestalis, Piracicaba, v. 42, n. 104, p. 483-490, 2014.

MENESES, V. A.; TRUGILHO, P. F.; CALEGARIO, N.; LEITE, H. G. Efeito da idade e do sítio na densidade básica e produção de massa seca de madeira em um clone do Eucalyptus urophylla. Scientia Forestalis, Piracicaba, v. 43, n. 105, p. 101-116, 2015.

MIRANDA, M. C.; CASTELO, P. A. R.; MIRANDA, D. L. C.; RONDON, E. V. Propriedades físicas e mecânicas da madeira de Parkia gigantocarpa Ducke. Ciência da Madeira, Pelotas, v. 3, n. 2, p. 55-65, 2012.

NASCIMENTO, C. C; GARCIA, J. N.; DIÁZ, M. D. P. Agrupamento de espécies madeireiras da Amazônia em função da densidade básica e propriedades mecânicas. Madera y Bosques, México, v. 3, n. 1, p. 33-52, 1997.

OLIVEIRA, J. T. S.; SILVA, J. C. Variação radial da retratibilidade e densidade básica da madeira de Eucalyptus saligna Sm. Revista Árvore, Viçosa, v. 27, n. 3, p. 381-385, 2003.

OLIVEIRA, J. T. S.; TOMAZELLO FILHO, M.; FIEDLER, N. C. Avaliação da retratibilidade da madeira de sete espécies de Eucalyptus. Revista Árvore, Viçosa, v. 34, n. 5, p. 929-936, 2010.

PANSHIN, A. J.; DE ZEEUW, C. Textbook of wood technology. 4.ed. New York: Mc-Graw-Hill, 1980. 736 p.

QUEIROZ, J. A. L.; MACHADO, S. A. Potencial da utilização madeireira de espécies florestais de várzea no município de Mazagão no estado do Amapá. Floresta, Curitiba, v. 37, n. 2, p. 293- 302, 2007.

SANTOS, V. F; TARDIN, A. T. Projeto zoneamento ecológico-econômico do setor costeiro estuarino do Estado do Amapá: diagnóstico socioambiental, relatório técnico de solos. Macapá: IEPA, 2003. 22 p. 
Araújo et al. - Propriedades físicas da madeira de Calycophyllum spruceanum Benth.

em função do diâmetro e da posição (base e topo) no fuste

SERPA, P. N.; VITAL, B. R.; LUCIA, R. M. D.; PIMENTA, A. S. Avaliação de algumas propriedades da madeira de Eucalyptus grandis, Eucalyptus saligna e Pinus elliottii. Revista Árvore, Viçosa, v. 27, n. 5, p. 723-733, 2003.

SETTE JR., C. A.; OLIVEIRA, I. R.; TOMAZELLO FILHO, M.; YAMAJI, F. M.; LACLAU, J. P. Efeito da idade e posição de amostragem na densidade e características anatômicas da madeira de Eucalyptus grandis. Revista Árvore, Viçosa, v. 36, n. 6, p. 1183-1190, 2012.

SILVA, J. C.; OLIVEIRA, J. T. S. Avaliação das propriedades higroscópicas da madeira de Eucalyptus saligna Sm., em diferentes condições de umidade relativa do ar. Revista Árvore, Viçosa, v. 27, n. 2, p. 233-239, 2003.

SILVEIRA, L. H. C.; REZENDE, A. V.; VALE, A. T. Teor de umidade e densidade básica da madeira de nove espécies comerciais amazônicas. Acta Amazônica, Manaus, v. 43, n. 2, p. 179-184, 2013.

SOTELO MONTES, C.; HERNÁNDEZ, R. E.; BEAULIEU, J. Radial variation in wood density and correlations with growth of Calycophyllum spruceanum at an early age in the Peruvian Amazon. Wood and Fiber science, Madison, v. 39, n. 3, p. 377-387, 2007.

SOTELO MONTES, C.; HERNÁNDEZ, R. E.; BEAULIEU, J.; WEBER, J. C. Genetic variation and correlations between growth and wood density of Calycophyllum spruceanum at an early age in the Peruvian Amazon. Silvae Genetica, Frankfurt, n. 55, p. 217-228, 2006.

SOTELO MONTES, C.; VIDAURRE, H.; WEBER, J. C. Variation in stem-growth and branch-wood traits among provenances of Calycophyllum spruceanum Benth. from the Peruvian Amazon. New Forests, Amsterdam, v. 26, n. 1, p. 1-16, 2003.

STATSOFT. Statistica (data analysis software system), version 10. StatSoft, Inc., 2012.

THIBAUT, B.; GRIL, J.; FOURNIER, M. Mechanics of wood and trees: some new highlights for an old story. Comptes Rendus de l’Académie des Sciences - Series IIB - Mechanics. v. 329, n. 9, p. 701-716, 2001.

TOMAZELLO FILHO, M. Variação radial da densidade básica e da estrutura anatômica da madeira do Eucalyptus saligna e E. grandis. IPEF, São Paulo, n. 29, p. 37-45, 1985.

TRAUTENMÜLLER, J. W.; BALBINOT, R.; BORELLA, J.; TREVISAN, R.; BALESTRIN, D.; VENDRUSCOLO, R.; SABADINI, A. M. Variação longitudinal da massa específica básica da madeira de Cordia americana e Alchornea triplinervia. Ciência Rural, Santa Maria, v. 44, n. 5, p. 817-821, 2014.

VALENTE, B. M. D. R. T.; EVANGELISTA, W. V.; SILVA, J. D. C.; LUCIA, R. M. D. Variabilidade radial e longitudinal das propriedades físicas e anatômicas da madeira de angico-vermelho. Scientia Forestalis, Piracicaba, v. 41, n. 100, p. 485-496, 2013.

VIDAURRE, G.; LOMBARDI, L. R.; OLIVEIRA, J. T. S.; ARANTES, M. D. C. Lenho juvenil e adulto e as propriedades da madeira. Floresta e Ambiente, Seropédica, v. 18, n. 4, p. 469-480, 2011.

WEBER, J. C.; SOTELO MONTES, C. Variation and correlations among stem growth and wood traits of Calycophyllum spruceanum Benth. from the Peruvian Amazon. Silvae Genetica, v. 54, n. 1, p. 31-41, 2005.

WOODCOCK, D. W. Wood specific gravity of trees and forest types in the southern Peruvian Amazon. Acta Amazônica, Manaus, v. 30, n. 4, p. 589-599, 2000.

ZENID, G. J. Madeira: uso sustentável na construção civil. São Paulo: Instituto de Pesquisas Tecnológicas, 2009. 103 p.

Recebido em 06/08/2015

Aceito para publicação em 01/03/2016 